
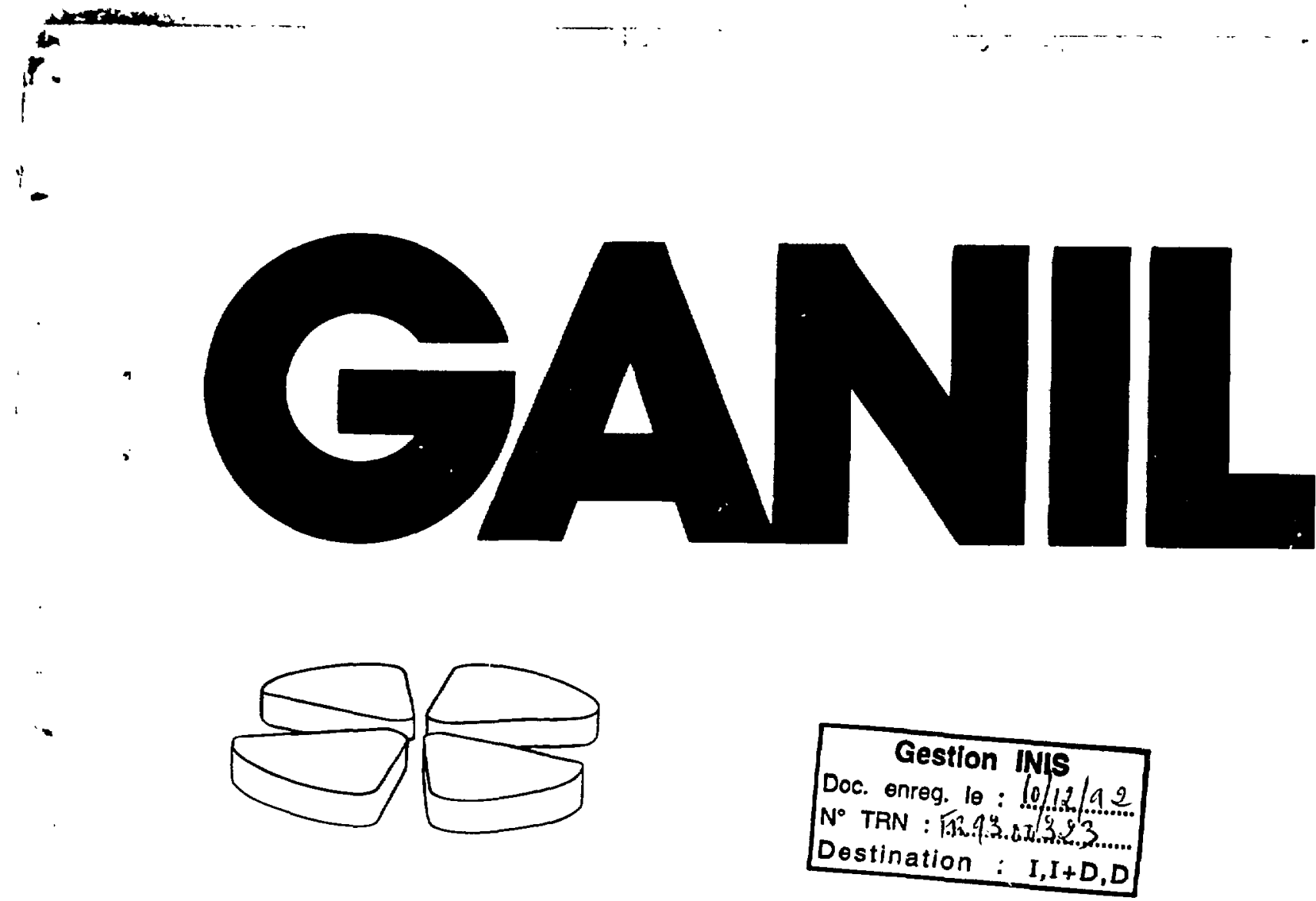

Simulating the Langevin force by simple noise in nuclear one-body dynamics *

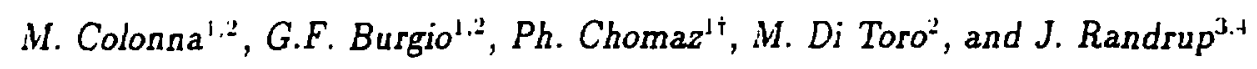

1) GANIL, B.P. 5027, F-14021 Caen Cedex, France

2) LNS, Viale Andrea Doria, Catania, Italy

3) Nuclear Science Division, Lawrence Berkeley Laboratory

University of California, Berkeley, California 94720, USA

1) GSI, Postfach 1105 52, D-6100 Darmstadt 11, Germany

GANIL P 9224

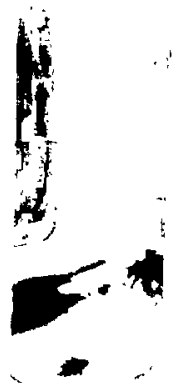




\title{
Simulating the Langevin force by simple noise in nuclear one-body dynamics *
}

\author{
M. Colonna ${ }^{1.2}$, G.F. Burgio ${ }^{1,2}$, Ph. Chomaz ${ }^{1 \dagger}$, M. Di Toro ${ }^{2}$, and J. Randrup ${ }^{3.1}$ \\ 1) GANIL, B.P. 5027, F-14021 Caen Cedex, France \\ $\left.{ }^{2}\right)$ LNS, Viale Andrea Doria, Catania, Italy \\ 3) Nuclear Science Division, Lawrence Berkeley Laboratory \\ University of California, Berkeley, California 94720, USA \\ ') GSI, Postfach 1105 52, D-6100 Darmstadt 11, Germany
}

October 26, 1992

\begin{abstract}
:
For the purpose of addressing catastrophic phenomena in nuclear dynamics, we explore the possibility of simulating the stochastic part of the collision integral in the Boltzmann-Langevin model by the numerical noise associated with the finite number of test particles in the ordinary $B U U$ treatment. Considering idealized twodimensional matter, for which it is practical to simulate the Boltzmann-Langevin equation directly, we demonstrate that the number of test-particles per nucleon can be adjusted so that the corresponding $B U U$ calculation yields a good reproduction of the spontaneous clusterization occurring inside the spinodal region. This approximate method may therefore provide a relatively easy way to introduce meaningful fluctuations in simulations of unstable nuclear dynamics.
\end{abstract}

- This work was supported in part by the Director, Office of Energy Research, Office of High Energy and Nuclear Physics, Nuclear Physics Division of the U.S. Department of Energy under Contract No. DE-AC03-76SF00098.

† On leave from Division de Physique Theorique, Institut de Physique Nucleaire, F-91406 Orsay Cedex, France. 


\section{Introduction}

Intense experimental and theoretical efforts are currently being directed at understanding the mechanisms responsible for the observed production of complex fragments in heavy-ion collisions. In recent years, intermediate-energy heavy-ion collisions have been studied extensively with $B U U$-type models, in which the collisionless Vlasov equation for the reduced one-body phase-space density $f(\mathbf{r}, \mathbf{p})$ is augmented by a Pauli-blocked Boltzmann collision term of the Uehling-Uhlenbeck form [1]. The resulting $B U U$ equation describes the average one-body density and is suited for the description of one-body observables, such as inclusive particle spectra. Models of this class have been quite successful in describing the structure of the equilibrated primary sources formed during the first stage of strongly damped and (incomplete) fusion reactions $[2]$.

However this mean-trajectory approach cannot provide a description of phenomena exhibiting large fluctuations or instabilities, such as multifragmentation processes. In such cases, a fully dynamical picture is essential for predicting particular space geometries in fragment production which should be ultimately related to fundamental properties of nuclear interactions in the nuclear medium. Thus there is a clear need for extending the usual mean-trajectory transport models so as to include fluctuations.

Generally speaking, in order to describe the formation of many fragments one should have to solve the full many-body problem, which is impractical. However, alternative avenues considering ensembles of one-body densities have been investigated $\{3,4,5,6]$. These approaches are equivalent to the so-called Boltzmann-Langevin model $(B L)$ in which the stochastic part of the two-body interaction is added to the mean $B U U$ evolution. In particular, using an exact lattice simulation of the $B L$ equation, it was shown [7] that in presence of instabilities, the fluctuations coming from the stochastic part of the collision integral are propagated and amplified by the mean field leading finally to the formation of clusters. Up to now this approach has been applied only to an idealized two-dimensional system [7], and it appears to be unfeasible to treat a three-dimensional scenario, such as a nuclear collision, because of the large amount of computational effort required.

We have therefore investigated the possibility of replacing the (usually complicated) physical fluctuations by a simpler noise. The main idea is that the dynamics of very unstable systems may be less sensitive to the particular nature of the fluctuations, because the most unstable modes will quickly dominate. Specifically, we study the possibility of replacing the physical fluctuations arising from the collision integral by the numerical noise present when the ordinary $B U U$ model is solved by the test-particle method, as is most commonly done. From a practical point of view, such a simplified approach would allow us to perform approximative calculations for realistic three-dimensional processes. 


\section{The Boltzmann-Langevin model}

The Boltzmann-Langevin equation can be written on compact form as follows,

$$
\dot{f} \equiv \frac{\partial f}{\partial t}+\{f, H[f]\}=I[f]=\bar{I}[f]+\delta I[f],
$$

where $f(\mathbf{r}, \mathbf{p}, t)$ is the one-body phase-space density. On the left-hand side, $H[f](\mathbf{r}, \mathbf{p})$ is the self-consistent effective single-particle Hamiltonian governing the collisionless Vlasov evolution. One the right-hand side, $I[f]$ denotes the effect of the residual two-body collisions, which can be decomposed into an average effect, $\bar{I}[f]$, and the fluctuating part $\delta I[f]$. The standard $B U U$ treatment retains only the average term $\bar{I}[f]$ and therefore produces a single dynamical trajectory for $f(\mathbf{r}, \mathbf{p})$. The fluctuating collision term $\delta I[f]$ acts like a random "kick"on the one-body density and may therefore produce branchings of the dynamics, resulting in a bundle of different histories.

The physical origin of the fluctuations can be understood as follows [8]: the actual number $\mathcal{N}$ of elementary collisions has a Poisson distribution characterized by its mean value $\bar{N}$ and so has the variance $\sigma_{i}^{2}=\bar{N}$. The average part of the collision integral, $\bar{I} \mid f]$, expresses the evolution correspondin $;$ r to putting $N=\bar{N}$ for all such elementary processes, while the fluctuating part accounts for the stochastic remainder $\delta N=N-\bar{N}$.

In order to gain insight into the growth of fluctuations we start from linear response theory, which is valid for small fluctuations and therefore is well suited for describing the early evolution of the system. Let us denote by $\nu$ a particular unstable mode of the $B U U$ dynamics and let its associated imaginary energy be $E_{\nu}=i \hbar / t_{\nu}$. Looking at the linear response to the Boltzmann-Langevin evolution, it is possible to demonstrate [9] that early on the average density fluctuation $\sigma_{\nu}^{2}$ in the unstable mode $\nu$ satisfies an equation of motion of the form,

$$
\frac{d}{d t} \sigma_{\nu}^{2} \approx 2 D_{\nu}+\frac{2}{t_{\nu}} \sigma_{\nu},
$$

where $D_{\nu}$ is the source term arising from the stochastic nature of the collision integral. It can be expressed as follows [9],

$$
2 D_{\nu}=\int \frac{d \mathbf{r} d \mathbf{p}}{h^{2}} \int \frac{d \mathbf{r}^{\prime} d \mathbf{p}^{\prime}}{h^{2}} F_{\nu}(\mathbf{r}, \mathbf{p}) F_{\nu}^{\star}\left(\mathbf{r}^{\prime}, \mathbf{p}^{\prime}\right) \alpha\left(\mathbf{r}, \mathbf{p} ; \mathbf{r}^{\prime}, \mathbf{p}^{\prime}\right),
$$

where $\alpha\left(\mathbf{r}, \mathbf{p} ; \mathbf{r}^{\prime}, \mathbf{p}^{\prime}\right)=\partial / \partial t \prec \delta f(\mathbf{r}, \mathbf{p}) \delta f\left(\mathbf{r}^{\prime}, \mathbf{p}^{\prime}\right) \succ_{1=v}$ is the early growth rate of the correlation function and $F_{\nu}(\mathbf{r}, \mathbf{p})$ is the eigenmode associated with the unstable mode.

It follows that the fluctuations of the mode $\nu$ exhibit the following behaviour in time,

$$
\sigma_{\nu}^{2}(t) \approx D_{\nu} t_{\nu}\left(\mathrm{e}^{2 t / l_{\nu}}-1\right)+\sigma_{\nu}^{2}(0) \mathrm{e}^{2 t / l_{\nu}},
$$

where $\sigma_{\nu}^{2}(0)$ is the initial fluctuation. This result shows that the terms $D_{\nu} t_{\nu}$ and $\sigma_{\nu}^{2}(0)$ play similar roles in the evolution. Therefore, it may be possible to replace the physical source term $D_{\nu}$ by a suitable initial noise $\sigma_{\nu}^{2}(0)$ determined by the relation

$$
\sigma_{\nu}^{2}(0)=D_{\nu} t_{\nu}
$$

This is the idea what we will now test in a simplified scenario. 


\section{Test in an idealized system}

In order to make a first quantitative test of the idea descibed above, we have considered a gas of Fermions situated on a two-dimensional torus with side lengths equal to $L_{x}=63 \mathrm{fm}$ and $L_{y}=21 \mathrm{fm}$. For the effective one-body field we employ a simplified Skyrme interaction,

$$
U(x)=A \frac{\bar{\rho}(x)}{\rho_{0}}+B\left(\frac{\bar{\rho}(x)}{\rho_{0}}\right)^{2},
$$

with $A=-100.3 \mathrm{MeV}$ and $B=48 \mathrm{MeV}$. Moreover, $\rho_{U}$ is the saturation density and $\bar{\rho}(x)$ is the average of the density $\rho(x, y)$ with respect to the transverse direction $y$ and folded in the $x$ direction with a Gaussian of width $a=0.87 \mathrm{fni}$. Since the effective field $U$ then depends on $x$ only, the same holds for those modes that can be excited, and this simplifies the analysis considerably. The folding simulates the finite range of the interaction which introduces a natural cutoff scale for the unstable modes. In determining the parameters of $U$, we have sought to mimic standard threedimensional matter. Thus we have required a Fermi momentum of $P_{F}=260 \mathrm{MeV} / \mathrm{c}$, and a binding energy of $16 \mathrm{MeV}$ per nucleon, and that a density doubling leads to approximately zero binding (corresponding to a coinpressibility modulus of $K \approx 300$ $\mathrm{MeV}$ for a calculation in three dimensions). The in-medium "cross-section" was taken as $2.4 \mathrm{fm}$, corresponding to a interaction radius of $1.2 \mathrm{fm}$. In the present idealized two-dimensional system, the saturation density is $\rho_{0}=0.55 \mathrm{fm}^{-2}$. In order to ensure that the system is initially situated inside the spinodal region, we prepare the system to have a uniform density equal to half the normal density, $\rho(x, y, t=0)=\frac{1}{2} \rho_{0}$, and having a temperature of $T=3 \mathrm{MeV}$. This physical scenario is similar to what was considered in ref. [7].

\subsection{Lattice simulation}

Recently a lattice simulation of the $B L$ evolution has been developed $[7,8,10]$. In this method the stochastic part of the collision number, $\delta N\left(12 ; 1^{\prime} 2^{\prime}\right)$ (see above), is simulated directly on a lattice in phase space. The size of the phase-space cells must be of the order $h^{2}$ in order to simulate the extension of an elementary quantum state and we have used $\Delta x=3 \mathrm{fm}$ and $\Delta p=120 \mathrm{MeV} / \mathrm{c}$. The mean-field evolution is treated by means of a standard matrix technique. However, in order to achieve sufficient accuracy, a second finer grid is required and therefore smaller cells having $\delta x=\frac{1}{3} \mathrm{fm}$ and $\delta p=40 \mathrm{MeV} / \mathrm{c}$ are employed. The transformations between these two scales are described in ref. [7]. We first focus on the evolution of the spatial density of the system, which is displayed in fig. 1a. Initially the system has a uniform density, but soon the fluctuations break this translational symmetry. Subsequently the fluctuations are rapidly amplified by the action of the effective one-body field, thus leading towards fragment formation. (Of course real fragments do not form, because the size of the torus is kept fixed so the system cannot expand.)

It is instructive to perform a Fourier analysis of the density for the considered system, $\rho(x)$. Indeed, in the present simplified scenario, the eigenmodes of the density are plane waves, characterized by a wave number $k$. The amplitude associated with 


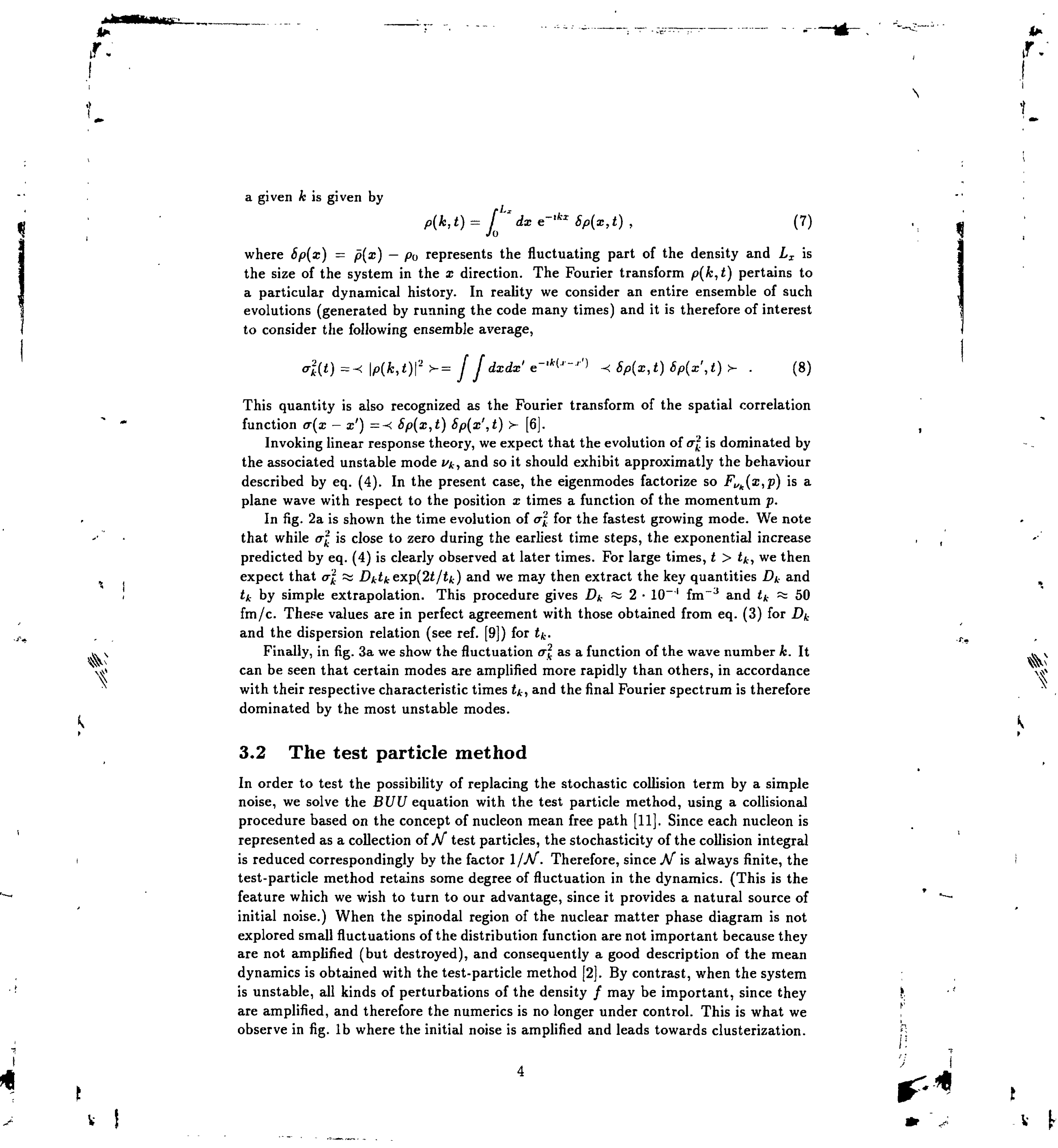


In this situation our idea is to carefully adjust the numerical fluctuations (i.e. the number of test particles $\mathcal{N}$ ) so as to simulate the physical fluctuations, which are well accounted in the lattice calculations (see ref. [8] for a detailed discussion).

Since the perturbation of the density introduced by using a finite number of test particles is of statistical nature, it may be calculated in a simple manner. Thus the number of test particles $n$ present in a volume $V$ fluctuates with a characteristic dispersion $\sigma_{n}^{2}=\bar{n}$, where $\bar{n}=\rho V \mathcal{N}$ is the mean number of test particles in $V$, which is fixed by the specified value of the initial density. Consequently the relative fluctuation of the density $\rho$ is $\sigma_{\rho} / \rho=1 / \sqrt{\bar{n}}$, or

$$
\sigma_{\rho}^{2}=\frac{\rho^{2}}{\bar{n}}=\frac{n}{V^{2} \mathcal{N}}
$$

The corresponding Fourier coefficient ( 8 ) is then independent of $k$,

$$
\sigma_{k}^{2}=\frac{L_{x}}{L_{y}} \frac{\rho}{\mathcal{N}}
$$

Thus, the numerical noise $\sigma_{k}^{2}$ scales as $1 / \mathcal{N}$, and so it is possible to choose the number of test particles $\mathcal{N}$ so as to reproduce the required magnitude of the physical source term $D_{k}$, for a particular value of the wave number $k$. Indeed, since in the $B L$ lattice calculation we have $\sigma_{k}^{2}(0)=0$, it follows from eq. (4) that asymptotically (i.e. for $t \gg t_{k}$ ) the magnitude of the fluctuations is determined by $D_{k} t_{k}$, whereas it is given by $D_{k} t_{k} / \mathcal{N}+\sigma_{k}^{2}(0)$ in the $B U U$ test-particle simulation. Therefore, the matching of those two asymptotic evolutions yields the condition

$$
(\mathcal{N}-1) D_{k} t_{k}=\mathcal{N} \sigma_{k}^{2}(0)=\frac{L_{x}}{L_{y}} \rho,
$$

which determines the number of test particles $\mathcal{N}$. Inserting the values determined above for $D_{k}$ and $t_{k}$, together with $L_{x}, L_{y}$, and $\rho$, we find $\mathcal{N} \approx 83$ for the fastest mode (the main uncertainty coming from the relatively inaccurate determination of the diffusion coefficient $D_{k}$ ).

We have then performed $B U U$ calculations using a range of values for $\mathcal{N}$ and compared the results with the corresponding lattice calculation. The time evolution of the fastest mode is shown in fig. 2 , in addition to the corresponding $B L$ result. The results are seen to bear out our expectation that the time evolutions follow the form given in eq. (4). Moreover, we observe that the lattice calculation is indeed well reproduced by the $B U U$ simulation when the value $\mathcal{N}=90$ is employed, which is in excellent agreement with the above result $\mathcal{N} \approx 83$.

Figure 1b shows the evolution of one particular density distribution (calculated with the optimal value $\mathcal{N}=90$ ), and it is seen that the density irregularities quickly attain the same magnitude as those of the lattice calculation.

In fig. 3 we show the quantity $\sigma_{k}^{2}$ as a function of the wave number $k$ for different times. We observe that not only are the time evolutions nearly the same, when comparing the $B L$ lattice calculation and the $B U U$ test-particle method, but also the spectral shape of the fluctuations are remarkably similar. This feature is very 
important for the viability of the method. The similarity of the spectral shapes arises from the fact that the product $D_{k} t_{k}$ depends only relatively weakly on $k$, in the neighborhood of the fastest mode, so that the speciral profile depends primarily on the amplification times $t_{k}$ which are the same in the two treatments.

\section{Discussion}

We have shown that when one is interested in the dynamics of very unstable systems the specific character of the fluctuations may not play a decisive role in the catastrophic evolution, provided the overall magnitude of the fluctuations is suitably adjusted. This is because the various unstable modes, once they have been agitated, are amplified exponentially so that the most unstable ones will tend to quickly dominate. In the present context, the magnitude of the imposed noise is set by the numerical parameter $\mathcal{N}$, the number of test particles per nucleon, which is determined by the relation (11). In the idealized two-dimensional case considered here, this number is around $\mathcal{N} \approx 90$, which is close to the value $\mathcal{N}=83$ pertaining to a full two-dimensional situation. We are presently in the process determining the value pertaining to three dimensions.

It should be noted that the source term $D_{k}$ depends on the phase-space density $f(\mathbf{r}, \mathbf{p})$. For example, at relatively moderate excitations it is proportional to the square of the temperature. Consequently, the optimal value of $\mathcal{N}$, as given by eq. (11) will depend on the the process considered and should therefore be carefully adjusted for the particular scenario under study. This feature is particularly relevant in connection with the very recent calculations showing various exotic structures in central symmetric collisions $[12,13,15,14,16]$, since these geometries depend sensitively on the time scale for the cluster formation, and hence the results will depend qualitatively on the particular value of $\mathcal{N}$ employed.

The advantage of the proposed test-particle method is that it makes it practically possible to perform meaningful three-dimensional simulations of nuclear collisions, a task that is presently beyond feasibility for the exact Boltzmann-Langevin model. However, we wish to emphasize that the simple method explored here should only be regarded as an approximate method for obtaining physically reasonable simulation of nuclear catastrophic processes using the standard $B U U$ model.

Finally, it should be mentioned that the two-body collision integral is not the only source of fluctuations in nuclear dynamics. This fact is perhaps most evident at lower energies where the Pauli blocking renders the collision integral ineffectual, and in fact the fluctuations observed for damped reactions have been largely understood as associated with the random character of the individual nucleon transfers.[17] A suggestion is to introduce a fluctuation in the effective field, $\delta H$, which can be done already at the level of the Vlasov equation [18]. Hopefully, by confronting carefu] calculations based on well-controlled approximations with experimental data, it will ultimately be possible to ascertain the relative importance of the various possible mechanisms.

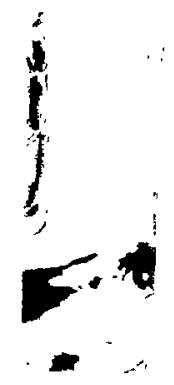




\section{References}

[1] G.F. Bertsch and S. Das Gupta, Phys. Rep. 160 (1988) 190

[2] M. Colonna et al, Phys. Lett. B283 (1992) 180; M. Colonna, N. Colonna, A. Bonasera, and M. Di Toro, Nucl. Phys. A541 (1992) 295

[3] W. Bauer, G.F. Bertsch, and S. Das Gupta, Phys. Rev. Lett. 58 (1987) 863

[4] S. Ayik and C. Gregoire, Phys. Lett. B212 (1988) 269; Nucl. Phys. A513 (1990) 187

[5] E. Suraud, S. Ayik, M. Belkacem, and J. Stryjewski, Nucl. Phys. A (in press)

[6] J. Randrup and B. Remaud, Nucl. Phys. A514 (1990) 339

[7] G.F. Burgio, Ph. Chomaz, and J. Randrup, Phys. Rev. Lett. 69 (1992) 885

[8] G.F. Burgio, Ph. Chomaz, and J. Randrup, Nucl. Phys. A529 (1991) 157

[9] Ph. Chomaz, M. Colonna, and J. Randrup, in preparation; M. Colonna, G.F. Burgio, and $\mathrm{Ph}$. Chomaz, in preparation.

[10] Ph. Chomaz, G.F. Burgio, and J. Randrup, Phys. Lett. B254 (1991) 340

[11] A. Bonasera, G.F. Burgio, and M. Di Toro, Phys. Lett. B221 (1989) 233

[12] L.G. Moretto, Kin Tso, N. Colonna, and G.J.Wozniak, Phys. Rev. Lett. $69(1992) 1884$.

[13] W. Bauer, G.F. Bertsch, and H. Schulz, Phys. Rev. Lett. 69(1992)1888.

[14] B. Borderie, B. Remaud, M.F. Rivet, and F. Sebille, Preprint IPNO-DRE (Jul'92)

[15] D.H.E. Gross, Bao-An Li and A.R. De Angelis, Ann. Phys. 1(1992)467; Bao-An Li and D.H.E. Gross, HMI preprint (Sept'92), Nucl. Phys. A, in press.

[16] G. Batko and J. Randrup, LBL-32722 (Sep'92), subm. to Nucl. Phys. A

[1i] W.U. Schröder and J.R. Huizenga, Treatise on Heavy-Ion Science, Vol. 2, (Ed. D.A. Bromley), Plenum Press (New York), 1984, p. 115

[18] R. Balian and M. Veneroni, Ann. Phys. (N.Y.)135, 270(1981); R. Balian and M. Veneroni, subm. to Annals of Physics (1992). 
Figure 1: Density profiles.

The density profile $\bar{\rho}(x, t)$ associated with one particular trajectory at four different times $t$, for the $B L$ lattice calculation (part $a$ ) and for the $B U U$ test-particle simulation (part b).

Figure 2: Growth of the fluctuations.

The time evolution of the Fourier transform of the correlation function, $\sigma_{k}^{2}$, for the most unstable mode (which has node number $K=k L_{x} / 2 \pi=6$ ), for the $B L$ lattice calculation (part $a$ ) and the $B U U$ test-particle simulation with three different values of $\mathcal{N}$, the number of test-particles per nucleon (part $b$ ). This quantity is the variance of fluctuations having wave number equal to $k$.

Figure 3: Variance spectrum.

The variance $\sigma_{k}^{2}$ as a function of the mode number $K\left(=k L_{x} / 2 \pi\right)$, shown at a number of times $t$ for the $B L$ lattice calculation (part $a$ ) and for the $B U U$ test-particle simulation with $\mathcal{N}=90$ (part $b$ ). 


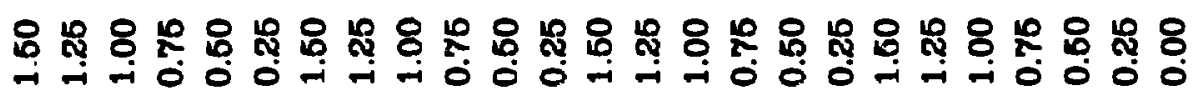

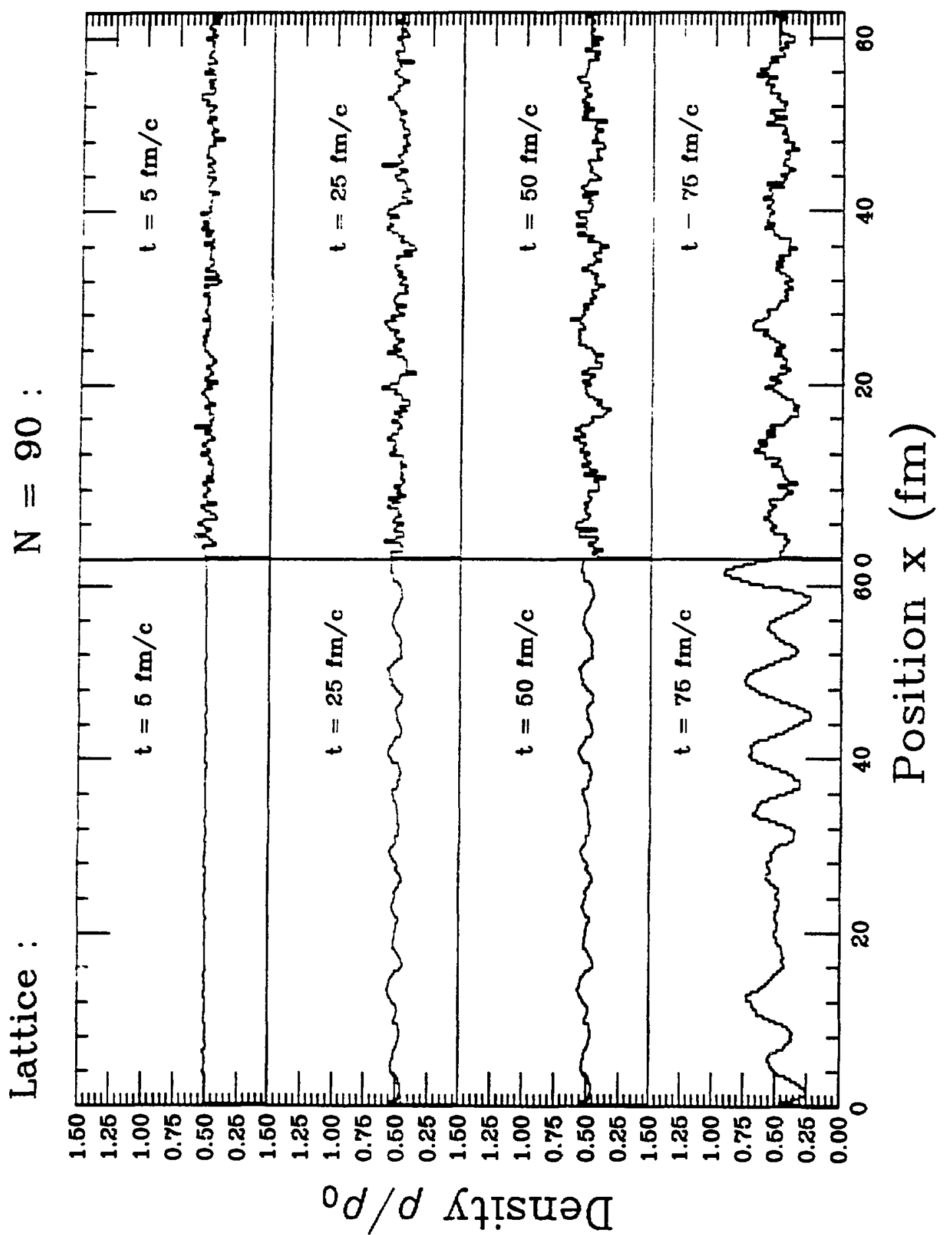

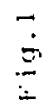




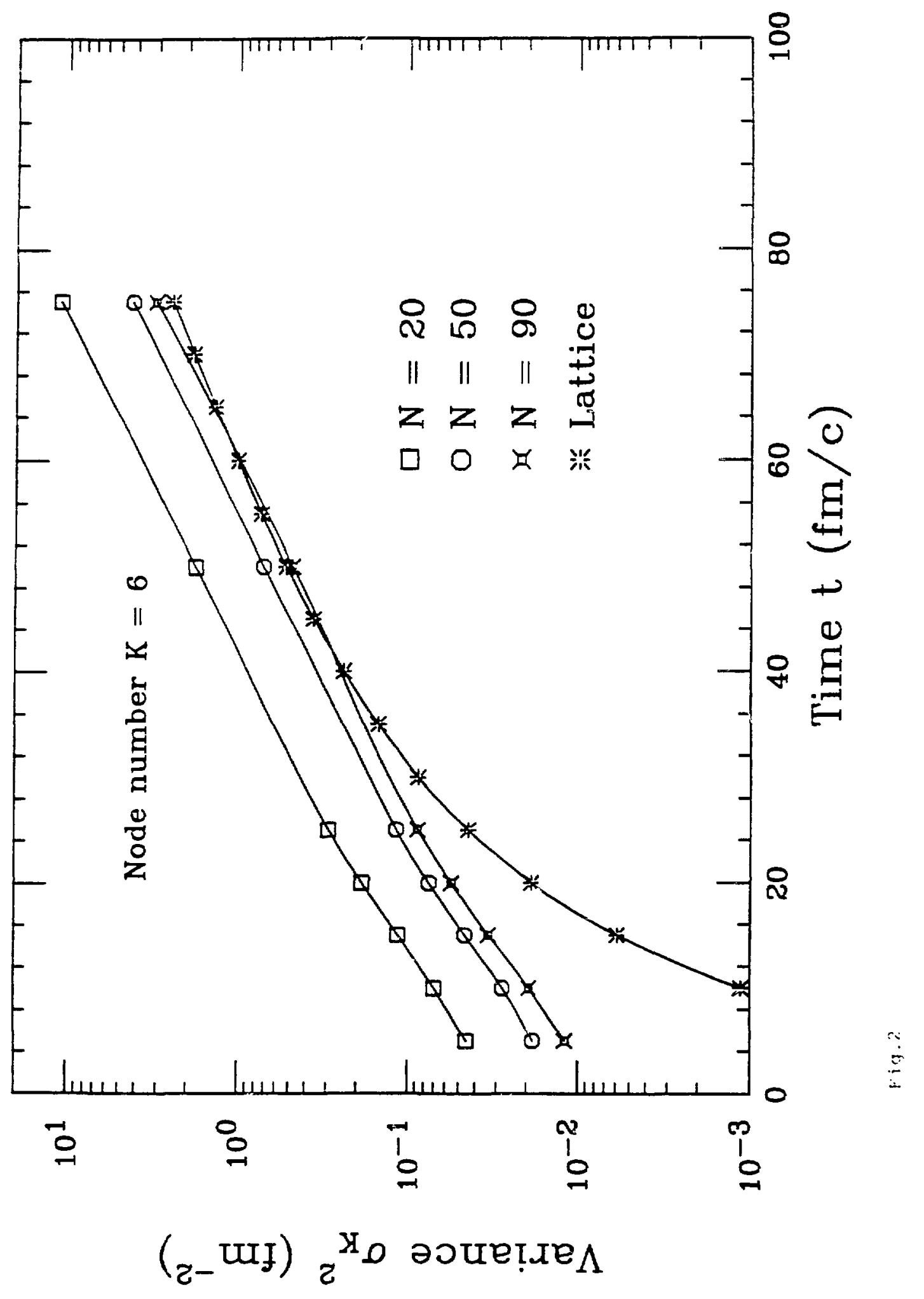




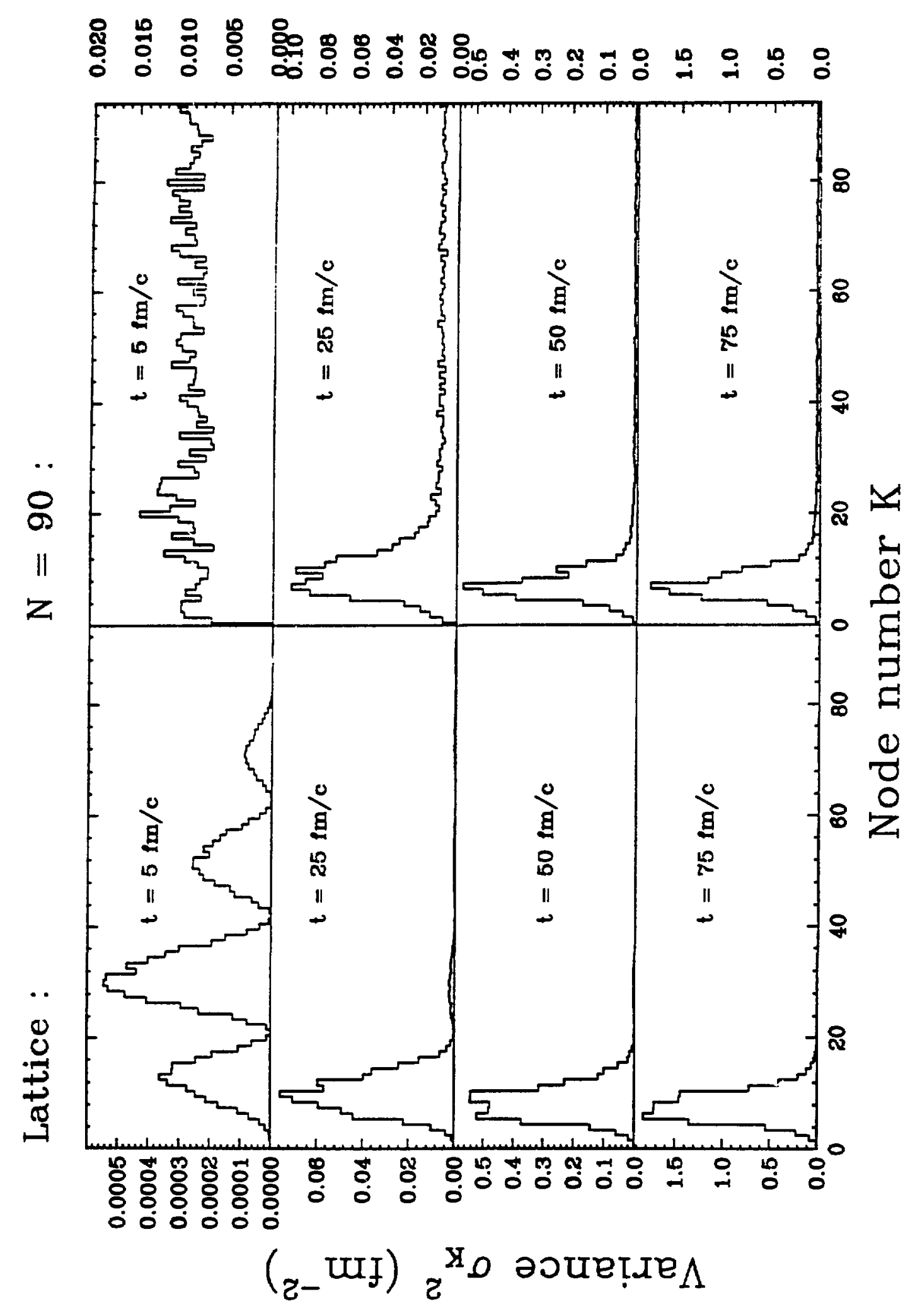

